\section{S3-Leitlinie Prostata als App}

Moderne Medizin braucht moderne Technik, diese sollte aber unkompliziert sein. Besitzer von iPhones können ab sofort auf die Prostata-Leitlinien für Urologen zugreifen. Die Broschüre „Das 1-2-3 der Prostata Leitlinien für PCA und BPS, Empfehlungen zur Prostatitis", welche die Kurzfassungen all dieser Leitlinien enthält, ist jetzt für iPhones in Form eines Apps verfügbar. Im deutschen AppStore ist „Das 1-2-3 der Prostata" zur Zeit der erste Treffer bei der Suche nach dem Stichwort Prostata. Einmaliges Herunterladen aus dem AppStore genügt, um das Kitteltaschenformat der Broschüre auch in der Hosentasche unterzubringen. Nach der Installation stehen die notwendigen Informationen zur Behandlung von Prostataerkrankungen immer zum Abruf bereit. Direkter Zugriff auf die drei Kapitel und eine hierarchische Darstellung der Inhalte erlauben den schnellen Zugriff auf die Informationen und machen das App zu einem praktischen Nachschlagewerk für Urologen.

Nach Informationen von Aviso, Berlin

\section{Zuverlässiger HIV-Schnelltest}

Der seit 2009 verfügbare HIVSchnelltest Determine ${ }^{\circledR} \mathrm{HIV}-1 / 2 \mathrm{Ag} /$ Ab Combo detektiert sowohl HIVAntikörper als auch das HIV-1 p24Antigen auf einem Teststreifen. In einer aktuellen schweizer Studie betrug die Sensitivität dieses HIVSchnelltests $100 \%$ : Keine der 300 chronisch HIV-infizierten Testpersonen wurde falsch negativ getestet. Bei keinem Mitglied der 69 HIVnegativen Studienteilnehmer in der Kontrollgruppe gab es ein falsch positives Resultat.

Nach Informationen von Alere, Köln

\title{
Histrelin: Kontinuierlich niedriges Testosteron
}

— Die Hormontherapie mit GnRH-Antagonisten hat in der individualisierten Therapie, vor allem bei Patienten im fortgeschrittenen metastasierten Stadium der Erkrankung einen wichtigen Stellenwert. ,Ziel der Hormontherapie ist es, dem androgensensitiven Tumor seine Nahrung zu entziehen", so Prof. Dr. Bob Djavan, New York. Um eine Krankheitsprogression zu verhindern, müssen die Testosteronwerte dauerhaft auf Kastrationsniveau (<20 ng/dl) gesenkt werden. „Der Cut-off von $50 \mathrm{ng} / \mathrm{dl}$ sei zu hoch angesetzt. Dies erläuterte der New Yorker Urologe anhand einer Studie [J Urol 2007; 178: 1290-5]: Bei einem kontinuierlichen Testosteronwert von weniger als $20 \mathrm{ng} / \mathrm{dl}$ betrug das mittlere Überleben von PCa-Patienten 106 Monate. Lag der Wert zwischen 20 und 50 ng/dl sank die Überlebenszeit auf 9o, und bei Durchbrüchen über $50 \mathrm{ng} / \mathrm{dl}$ auf 72 Monate.

Bereits solche vorübergehenden Anstiege der Serum-Testosteron-Konzentration, die Flare-Ups, können offenbar hormonsensi- tive Krebszellen reaktivieren. Bei der Therapie mit bisherigen $\mathrm{GnRH}$-Agonisten finden sich Durchbrüche der Testosteronwerte über $20 \mathrm{ng} / \mathrm{dl}$ bei über $55 \%$ der Patienten. Hier weist das Implantat mit dem retardierten GnRH-Agonisten Histrelin (Vantas ${ }^{\circledR}$ ) bessere Daten auf. Djavan berichtet: „So konnte in der Zulassungsstudie gezeigt werden, dass durchschnittlich $88 \%$ der Patienten ab der Woche vier nach Implantation kontinuierliche Testosteronwert von unter 20 ng/dl aufwiesen." Und dies konnte drei Jahre gehalten werden. Die Galenik des Implantats ermöglicht eine gleichmäßige Freisetzung über ein Jahr. Auch eine gute Verträglichkeit haben klinische Studien gezeigt. $\quad$ rfr

Satellitensymposium „Chancen, Nutzen und Erfolg - die PCa-Therapie heute“, im Rahmen des 62. Kongresses der Deutschen Gesellschaft für Urologie, Düsseldorf, 23. September 2010

Veranstalter: Orion Pharma, Hamburg

\section{Elektrotherapie mal zwei}

— Entscheidend bei der Behandlung einer weiblichen Stress-, Drang- und Mischinkontinenz ist die Stärkung der Selbstkontrolle. Die Kontraktionsfähigkeit gezielt zu trainieren, gilt daher als Schlüssel einer nicht-medikamentösen, nicht-invasiven Therapie. Ergänzend zur Physiotherapie haben sich die elektrische Muskelstimulation (EMS) des Beckenbodens sowie Biofeedback mittels Elektromyografie (EMG) bewährt. Beide Techniken wirken bei Hypo-, Hyper- oder Akontraktilität der Beckenbodenmuskulatur. Die Bio-Medical-Research-Gruppe (neurotech $^{\circledR}$ ) bietet mit CombiStim XP ${ }^{\circledR}$ ein Elektrotherapiegerät, das beide Verfahren vereint. Während Biofeedback insbesondere für Patientinnen geeignet ist, die ihre Beckenbodenmuskulatur noch schwach anspannen können, dient die EMS-Therapie dazu, die Kontraktilität starrer oder inaktiver Muskulatur durch elektrische Impulse zu reaktivieren sowie bei Dranginkontinenz zu beruhigen. Sinnvoll kann eine Kombination beider Verfahren sein, wenn noch oder bereits wieder eine gewisse Kontraktilität der Becken-

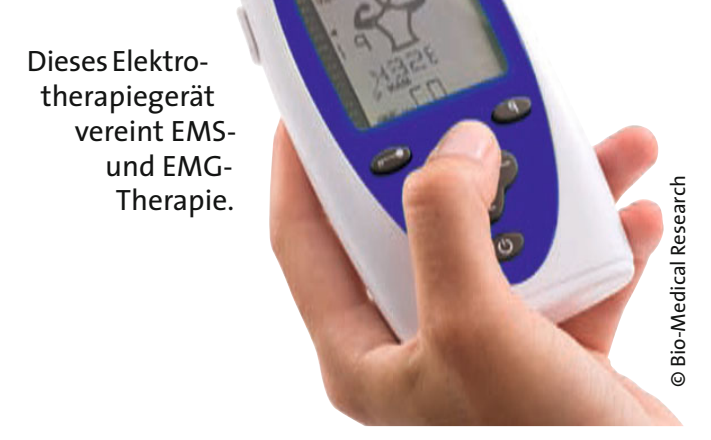

bodenmuskulatur gegeben ist. Bei der kombinierten Anwendung von Biofeedback und EMS wird eine gemessene Muskelanspannung bei Überschreitung eines definierten Schwellenwertes durch einen zusätzlichen Impuls verstärkt. Diese Form der Elektrotherapie heißt EMG-triggered Stimulation (ETS). Aktive und passive Muskelstärkung gehen somit Hand in Hand und steigern den Trainingseffekt.

Nach Informationen von

Bio-Medical Research, Salem 